\title{
Indicadores del impacto del cambio climático en la agricultura familiar andina colombiana
}

\author{
Indicators of the impact of climate change on andean family \\ agriculture in Colombia
}

\author{
Núñez, Jesús; Carvajal, Julio César; Mendoza, Omaira; Carrero, Darcy; \\ Editor Académico Prof. Dr. Ángel Sol-Sánchez
}

\author{
Jesús Núñez \\ jo.nunez@mail.udes.edu.co \\ Universidad de Santander (UDES), Campus Cúcuta, \\ Colombia. , Colombia \\ Julio César Carvajal \\ departamentodeeducacion@cucuta.udes.edu.co \\ Universidad de Santander (UDES), Campus Cúcuta, \\ Colombia., Colombia \\ Omaira Mendoza \\ itamendoza@hotmail.com \\ Universidad de Santander (UDES), Campus Cúcuta, \\ Colombia., Colombia \\ Darcy Carrero \\ darcycarrero@gmail.com \\ Universidad Nacional Experimental del Táchira \\ (UNET), Venezuela., Venezuela \\ Editor Académico Prof. Dr. Ángel Sol-Sánchez \\ Colegio Postgraduados, México
}

Revista Iberoamericana de Bioeconomía y Cambio Climático

Universidad Nacional Autónoma de Nicaragua, León, Nicaragua ISSN-e: 2410-7980

Periodicidad: Semestral

vol. 4, núm. 7, 2018

czuniga@ev.unanleon.edu.ni

Recepción: 15 Mayo 2018

Aprobación: 16 Julio 2018

URL: http://portal.amelica.org/ameli,

jatsRepo/394/3941754002/index.htm

DOI: https://doi.org/10.5377/ribcc.v4i7.6309

Autor de correspondencia: jo.nunez@mail.udes.edu.co
Resumen: Los modelos de evaluación de los impactos del cambio climático utilizados en la agricultura colombiana se han enfocado en cultivos tecnificados, sembrados en grandes superficies, obviando que el $67 \%$ de la producción nacional de alimentos provienen de pequeñas extensiones de policultivos cultivados, en su mayor parte, en suelos de ladera de la cordillera andina, por 3,5 millones de agricultores y que aportan el 57\% del empleo rural. El presente artículo se deriva de un análisis de la metodología empleada por los autores en el proyecto de investigación denominado "Impactos económicos, ambientales y sociales del cambio climático en el sector agropecuario del Municipio Bochalema, Departamento Norte de Santander, Colombia, financiado por la Universidad de Santander (UDES) y que abarca el período comprendido entre enero de 2017 y mayo de 2018. Después de analizar diferentes modelos de evaluación de impactos ambientales se seleccionó el modelo de Presión, Estado, Respuesta (PER) de la OCDE, previa adaptación a las características específicas de pequeñas unidades de producción agrícola de subsistencia. Se evidenció la multidimensionalidad y complejidad de los factores relacionados con el clima, la producción, los recursos, las tecnologías, las familias y las políticas públicas, que obligan a la construcción de indicadores contextualizados a la agricultura familiar andina.

Palabras clave: Bio Indicadores, Cambio climático, Agricultura familiar JEL: Q01, Q51, Q54.

Abstract: The climate change impact assessment models used in Colombian agriculture have focused on technified crops, planted in large areas, ignoring the fact that $67 \%$ of national food production comes from small extensions of polycultures, most of which are grown on slopes of the Andean mountain range, by 3.5 million farmers and which provide $57 \%$ of rural employment. This article is derived from an analysis of the methodology used by the authors in the research project entitled "Economic, environmental and social impacts of climate change on the agricultural sector in the Municipality of Bochalema, Norte de Santander Department, Colombia, funded by the University of Santander (UDES) and covering the period from January 2017 to May 2018. After analyzing different models of environmental impact assessment, the OECD's Pressure, State, Response (PER) model was selected, after adaptation to the specific characteristics of small subsistence agricultural 
production units. The multidimensionality and complexity of factors related to climate, production, resources, technologies, families and public policies were highlighted, which require the construction of contextualized indicators for Andean family agriculture.

Keywords: Bio Indicators, Climate change, family farming JEL: Q01, Q51, Q54.

\section{INTRODUCCIÓN}

El cambio climático es una realidad que afecta lo global y encuentra en Colombia un espacio territorial de alta sensibilidad geográfica y social, hecho que amerita revisar profundamente las implicaciones sobre los sectores de la sociedad, el ambiente y la producción. Dentro de ellos el sector agropecuario, por ser un sistema con alta dependencia ambiental, acoge significativamente las variaciones graduales de las temperaturas y las precipitaciones sobre los recursos naturales, agrícolas, pecuarios y humanos. Esta complejidad de factores incidentes y receptores se evidencia en mayor grado en la cordillera andina por su diversidad geológica, ecosistémica, climática y antrópica que potencializa los impactos del cambio climático sobre lo rural y agropecuario. A los fines de revisar esta problemática en la agricultura familiar de los andes colombianos se delinearon las siguientes premisas de trabajo: 1) La región andina presenta alta vulnerabilidad al cambio climático; 2) La agricultura familiar es predominante en la producción andina; 3) Los cambios medioambientales de temperatura y precipitación incrementan la presión agrícola sobre los recursos de los ecosistemas andinos; 4) La evaluación del impacto del cambio climático en Colombia ha estado orientada, principalmente, a rubros agropecuarios tecnificados y comerciales, con poca atención a la agricultura familiar de subsistencia y; 5) Es urgente la construcción de modelos de evaluación con indicadores pertinentes y contextualizados para determinar los efectos del cambio climático en la agricultura familiar andina, la cual ocupa la mayor superficie en producción, es generadora de gran cantidad de empleos, resguarda la rica biodiversidad andina y garantiza la seguridad alimentaria de los colombianos.

\section{REVISIÓN DE LA LITERATURA}

\section{A. La necesidad de construir indicadores del cambio climático para la agricultura familiar andina}

La agricultura familiar andina, por su naturaleza particular, realiza actividades de apoyo a la reducción al cambio climático, una de ellas es la adopción de prácticas sostenibles en la interpretación y utilización de bioindicadores vegetales y animales e indicadores atmosféricos, propios del saber campesino; la plantación de árboles para la protección de suelos y cauces hídricos; la "siembra del agua" como práctica ancestral para la generación de nuevos nacimientos de agua; el fortalecimiento de la biodiversidad a través de la preservación de especies vegetales y animales (policultivos) y; la diversificación de la producción mediante el uso de recursos endógenos, entre otras; estas acciones contribuyen a aumentar la resiliencia, regeneración y capacidad de adaptación de agricultura familiar frente al cambio climático (Meza, 2014).

Notas DE AUTOR 
La cultura rural forjada alrededor de este tipo de agricultura, en la cual interactúan componentes religiosos, míticos, productivos, sociales y ambientales, le otorgan unas particulares especiales a considerar en la evaluación de los impactos ambientales derivados de las variaciones climáticas, desde unos referentes conceptuales, metodológicos y técnicos adaptados a sus realidades, como una herramienta para conocer e interpretar los efectos de este fenómeno sobre estos entornos específicos (Conesa, 2010; Canter, 1998; Perevochtchikova, 2013).

Debido al campo extenso de las disciplinas, donde la evaluación de los impactos ambientales, es utilizada se han derivado una gran diversidad de metodologías diseñadas para captar fehacientemente los impactos, en tiempo, espacio y recursos, que han producido las intervenciones sociales, culturales, económicas, tecnológicas y ambientales sobre un objeto de interés para la transformación, análisis y/o la generación de nuevo conocimiento (Organización Internacional del Trabajo -OIT-y Centro Interamericano para el Desarrollo del Conocimiento en la Formación Profesional -CINTERFOR-, 2011).

Es de aclarar, que aunque la generalidad de las definiciones encontradas en las referencias revisadas, sobre la evaluación de impactos ambientales, refieren a la determinación de los efectos en el corto y mediano plazo de políticas, programas o proyectos sobre sectores de la sociedad (Espinoza, 2002; Conesa, 2010; Canter, 1998) existen en ellas elementos comunes que caracterizan al impacto ambiental, como son las nociones de causalidad y efecto (Garmendia, et. al., 2005); es decir, la relación causa-efecto que se deriva de someter una situación particular inicial a una experiencia de intervención deliberada, planificada y sistemáticamente desarrollada.

Esta situación epistémica también se evidencia en las definiciones de Evaluación del Impacto Ambiental (EIA), como un instrumento técnico-administrativo de gestión ambiental para prever las implicaciones ambientales que un proyecto o acción puede ocasionar sobre el entorno natural (Ministerio de Vivienda, Ordenamiento Territorial y Medio Ambiente del Uruguay, 2016; Conesa, ob.cit.). La visión preliminar de las determinaciones de impacto ambiental sobre un medio físico-natural y social constituye una herramienta prospectiva útil para prevenir, mitigar o adaptar los desarrollos y actividades a parámetros ambientales permitidos de sostenibilidad (Buroz, 1998; Perevochtchikova, ob.cit.).

Ahora bien, al situarse en el campo de la evaluación del cambio climático es importante destacar que precisa de 4 conceptos básicos relacionados con el riesgo, a tener en cuenta: vulnerabilidad, exposición, mitigación y adaptación (Grupo Intergubernamental de Expertos sobre el Cambio Climático -IPCC-, 2014a).

El primero de ellos se refiere a la sensibilidad a riesgos altos y moderados de los ecosistemas naturales y humanos al cambio climático; el segundo, al nivel de protección que los recursos y personas tienen ante eventos extremos; el tercero, a las medidas técnicas-administrativas para disminuir los impactos ambientales y; el cuarto, a los mecanismos para que los ecosistemas y comunidades se adapten a condiciones emergentes de temperatura y precipitación dentro de los límites de supervivencia.

Las consideraciones del IPCC, plasmadas en el Quinto Informe del Grupo de Trabajo II (2014b), son útiles para guiar la evaluación de los impactos del cambio climático en el sector agropecuario al valorar los riesgos de los procesos económicos, socioculturales y sistemas naturales a los efectos de las variaciones climáticas en los ámbitos de la vulnerabilidad, exposición, mitigación y adaptación (Mussetta et al., 2016; Peralvo y López, 2010).

En los modelos más utilizados de evaluación del cambio climático se evidencia una tendencia a enmarcarlos dentro de la evaluación de vulnerabilidad al asumir que el cambio climático implica un riesgo sobre la sostenibilidad y vitalidad de los sistemas naturales y humanos (Departamento Nacional de Planeación (DNP), 2014; México Resiliente, 2013; Robles, 2016). Estos modelos están conformados, generalmente, por tres fases: Diagnóstico, Modelo Conceptual y Validación. El diagnóstico permite reconocer y comprender la importancia, magnitud del problema y la sensibilidad de los ecosistemas y poblaciones humanas ante los cambios ambientales; el modelo conceptual parte del conocimiento teórico y de la experiencia diagnosticada en el entorno para generar una explicación de causas-efectos-soluciones orientada a mitigar y adaptar los 
sistemas naturales y humanos a las variaciones ambientales y a eventos climáticos extremos y; la validación es poner a prueba el funcionamiento del modelo teórico en escenarios históricos de impactos y vulnerabilidades para determinar su eficiencia.

Específicamente, la medición de los impactos del cambio climático en la agricultura ha seguido dos enfoques metodológicos: el estructural y el espacial (Comisión Económica para América Latina y el Caribe - CEPAL-, 2015; Ordaz, et al., 2010). El primero mide los impactos de las variables climatológicas (temperatura y precipitación) en la función de producción (rendimientos, productividad, costos), de tal forma que puede modelar en diferentes escenarios temporales las tendencias del comportamiento del clima y de la producción. El segundo enfoque, el espacial, mide los efectos directos del cambio climático sobre unidades físicas determinadas (fincas, bosques, etc) al diferenciar patrones en la calidad y disponibilidad de los recursos agrologicos (calidad de los suelos, disponibilidad de agua, distribución de los cultivos). En la práctica, estos dos enfoques se utilizan de manera complementaria para obtener una visión integradora del impacto del cambio climático en la agricultura.

La comprensión e interpretación de la evaluación del impacto del cambio climático en la agricultura, desde múltiples perspectivas, -cuantitativas y cualitativas-, posibilitan la generación de modelos-soluciones más pertinentes y contextualizados como garantía de intervenciones efectivas en los escenarios ambientales, económicos y sociales donde se desarrollen. Indudablemente, la comprensión no se logra en su totalidad si se percibe desde pequeñas miradas; por lo que se debe mirar al otro y a sí mismo como un constructor de conocimiento (Carrero, 2016), al valorar y sistematizar el saber campesino en la formulación de los indicadores para la construcción de un modelo adaptado a sus contextos productivos y sociales (Núñez, 2005).

El uso de indicadores, como producto de la evaluación del impacto del cambio climático, es una de las herramientas eficaces a la hora de tomar decisiones para diseñar políticas, programas y proyectos de intervención (Yepes, 2012) orientados a mitigar y adaptar a la población y a los sistemas productivos a escenarios prospectivos de variaciones climatológicas en un territorio determinado (Rave, 2016). De manera tal, que los indicadores son puntos de referencia o comparación (Beltrán, 2005) que señalan (indican) información del desempeño de un proceso, sistema u organización a partir de información cuantitativa o cualitativa provenientes de datos recabados por percepciones, opiniones, mediciones y hechos, los cuales son sistematizados y analizados siguiendo metodologías científicas y técnicas para su construcción; buscando orientar el estado, condición de un proceso o fenómeno en relación con la sostenibilidad (Ramírez et al., 2008).

En el ámbito de los indicadores existen diferentes tipologías, algunas de las cuales se pueden agrupar en: indicadores de evaluación ambiental; indicadores de integración sectorial; indicadores de integración económica, entre otros (Manteiga, 2000; Organización para la Cooperación y el Desarrollo Económico - OCDE-, 2001), en función a los requerimientos de las disciplinas, campos de acción y propósito de la evaluación, por lo que es difícil enmarcarse dentro de una clasificación particular. En los modelos más utilizados de evaluación del cumplimiento de metas económicas, sociales y ambientales se construyen indicadores para cada ámbito específico con unidades de medidas adecuadas a su medición (Secretaría de Agricultura, Ganadería, Desarrollo Rural, Pesca y Alimentación (México) -SAGARPA-, 2016)

Cabe resaltar, que en Colombia algunos sistemas agrarios están más consolidados en materia de investigación agrometeorológica y en redes de observaciones meteorológicas por lo que tienen mayor acceso a pronósticos y predicciones. Sistemas agrarios, como el café, palma aceitera, caña de azúcar, cereales y arroz han tenido más avance con el uso de diversos modelos que permiten simular la disponibilidad hídrica, rendimientos potenciales, crecimiento del cultivo y respuesta a diversas alternativas de manejo. De otra parte, se encuentran los pequeños agricultores -de quienes depende el $67 \%$ de la producción nacional (Ministerio de Agricultura y Desarrollo Rural, 2014)- con poco, o ningún, acceso a la información agroclimática. Es por esto, que la incidencia negativa del comportamiento climático en Colombia, en la producción y rendimiento 
agrícola de los diversos cultivos, prevención y control de riesgos y desastres, dificulta la toma de decisiones de políticas públicas de apoyo para las economías campesinas andinas.

\section{B. Indicadores para la construcción de un modelo de evaluación del impacto del cambio climático en la agricultura familiar andina}

Es relevante señalar el interés de algunos autores en el estudio del efecto del cambio climático sobre pequeños productores agrícolas. Altieri y Nicholls (2008) describen los impactos del cambio climático en agricultores tradicionales y las características agroecológicas de los sistemas agrícolas indígenas, los cuales pueden ser útiles para el diseño de sistemas agrícolas e indicadores en los planes de adaptación. Asimismo, organizaciones advierten que los pequeños productores son los más vulnerables frente al cambio climático (Instituto de Hidrología, Meteorología y Estudios Ambientales -IDEAM- y el Fondo Financiero de Proyectos de Desarrollo-FONADE-, 2013).

Entre los modelos más utilizados para la evaluación del impacto ambiental se encuentra el denominado Presión-Estado-Respuesta (PER) (OCDE, ob.cit) que utiliza 3 categorías de indicadores: Presión: Intensidad de actividades humanas sobre los recursos; Estado: Condición que refiere a la afectación en que se encuentran los sistemas naturales, económicos y sociales y; Respuesta: Acciones públicas y privadas para mitigar o prevenir la presión de los factores generadores de cambio climático.

En el caso de la agricultura familiar andina el modelo PER podría ser adaptado para evaluar el impacto del cambio climático de la siguiente forma: Presión: Influencia de las actividades antrópicas sobre el entorno e intensidad de las variaciones de los factores climáticos (temperatura y precipitación); Estado: Efectos (negativos y positivos) del cambio climático en los sistemas productivos, sociales, económicos y ambientales del sector agropecuario y; Respuesta: Políticas, programas, proyectos y buenas prácticas de los agricultores (saberes campesinos) para mitigar y adaptar al sector agropecuario al cambio climático (Figura 1). El modelo PER adecuado a la pequeña agricultura quedaría así:

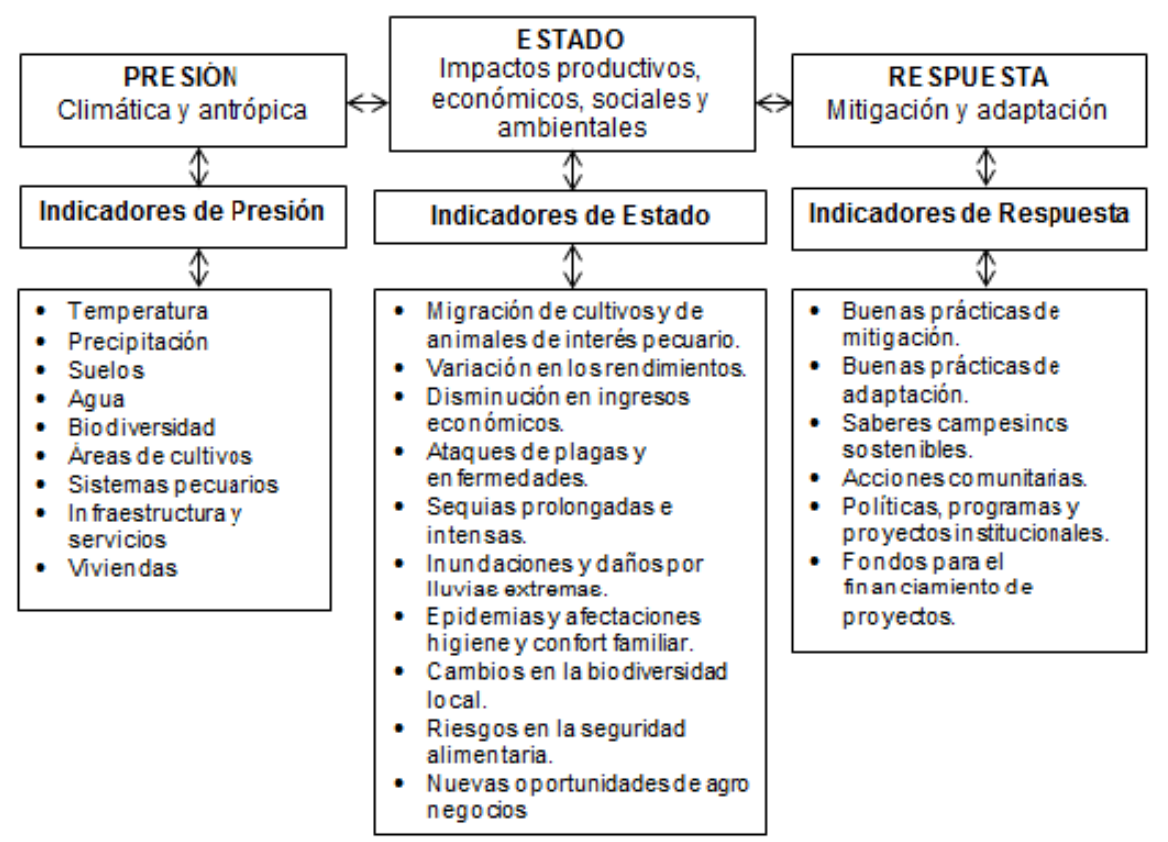

FIGURA 1.

Modelo PER, adaptado a la agricultura familiar andina Modelo PER (OCDE, 2001), adaptado por los autores (2018) 
Dentro de la adaptación del Modelo PER a la agricultura familiar andina se deben valorar como indicadores de presión sobre las unidades de producción familiar las variaciones de las temperaturas y de las precipitaciones en registros históricos de datos climatológicos y; la presión antrópica sobre los recursos edáficos e hídricos, la biodiversidad vegetal y animal, las superficies bajo cultivo, los sistemas de explotación pecuaria, las infraestructuras y servicios y los asientos residenciales de los agricultores en las fincas o centros poblados rurales.

Las pulsiones climatológicas y antrópicas sobre los contextos campesinos generan un conjunto de impactos, denominadas en el Modelo PER como Estado, las cuales pueden ser evaluadas considerando, entre muchos, los siguientes indicadores de estado: Las migraciones de cultivos y animales de interés pecuario por los cambios en las temperaturas, disponibilidad de agua, fertilidad de los suelos, calidad de los productos e incidencia de plagas y enfermedades; repercusiones en la productividad, rendimientos e ingresos económicos de los rubros destinados al mercado; los efectos de las sequias prolongadas y lluvias extremas sobre los recursos naturales, productivos y tecnológicos; las alteraciones en la biodiversidad de los agro ecosistemas por migración, extinción y resiliencia de especies de la flora y fauna local; los efectos sobre las familias campesinas relacionadas con insectos, vectores, enfermedades, higiene familiar y seguridad alimentaria y; nuevas oportunidades de agro negocios por las condiciones que se derivan del calor y disponibilidad de agua para el desarrollo de especies con demandas diferenciadas y potencialidades de desarrollo.

Finalmente, dentro de los indicadores de respuesta es importante sistematizar y valorar las acciones internas de los grupos sociales campesinos, como las buenas prácticas de mitigación (sombríos, reforestación de nacientes hídricas, "siembra del agua", preservación de la biodiversidad, diversificación de cultivos (policultivos), protección de los suelos, uso de abonos orgánicos, cubiertas vegetales, manejo del agua, etc) y de adaptación (incorporación de nuevos cultivos y especies animales, control y manejo de plagas, cuidado de la salud y alimentación familiar, entre otras) y los saberes de los campesinos sobre la utilización de bioindicadores vegetales, animales y atmosféricos en la interpretación ambiental y en las prácticas de uso de los recursos naturales, manejo de los sistemas productivos y de construcciones con tecnologías sostenibles. Asimismo, dentro de los indicadores de respuesta es importante evaluar las acciones externas desarrolladas por instituciones públicas y privadas (escuelas, alcaldías, gobernaciones, corporaciones de desarrollo, ministerios, universidades, empresas, etc) para abordar los problemas ocasionados por el cambio climático en el sector campesino.

\section{CONCLUSIONES}

Colombia depende en un $67 \%$ de su producción nacional de la pequeña agricultura, predominantemente asentada en la cordillera andina, una región vulnerable geológica, ecológica y socialmente a los impactos del cambio climático.

En los estudios realizados en el país para evaluar los impactos del cambio climático en la agricultura es evidente su interés en los sistemas tecnificados y comerciales de grandes extensiones, con escasa dedicación a las pequeñas superficies cultivadas por la mayoría de los productores agropecuarios.

Es urgente e importante evaluar los impactos del cambio climático en la agricultura familiar mediante un modelo pertinente y contextualizado de indicadores que considere y valore las particularidades de las unidades productivas agrícolas dentro de la cosmovisión de la cultura campesina andina.

El Modelo PER (Presión-Estado-Respuesta) de la OCDE es una herramienta sistémica que brinda un soporte metodológico apropiado para ser ajustado a la evaluación de los impactos del cambio climático en la agricultura familiar andina con indicadores económicos, ambientales, sociales imbricados en la cultura rural andina. 


\section{LiTERATURA CITADA}

Altieri, M.; Nicholls, C. (2008). Los impactos del cambio climático sobre las comunidades campesinas y de agricultores tradicionales y sus respuestas adaptativas. Revista de la Agroecología 3, 7-23. ISSN electrónico: 1989-4686.

Beltran, J. M. (2005). Indicadores de Gestión, Herramientas para lograr la competitividad (2 Edición ed.). Bogota: Panamericana.

Buroz Castillo, E. (1998). La gestión ambiental: marco de referencia para las evaluaciones de impacto ambiental. Caracas, Venezuela: Fundación Polar, 376.

Canter, L. (1998). Manual de Evaluación de Impacto Ambiental: Técnicas para la Elaboración de Estudios de Impacto. McGraw-Hill, Madrid, España.

Carrero, D. (2016). Representaciones sociales del agua en el contexto universitario. Carrera de Ingeniería Ambiental. Universidad Nacional Experimental del Táchira (UNET). Tesis de Doctorado, no publicada, Universidad Pedagógica Experimental Libertador (UPEL), Venezuela.

Comisión Económica para América Latina y el Caribe (CEPAL). (2015). El cambio climático y sus efectos en la biodiversidad en América Latina. Programa EUROCLIMA. . Naciones Unidas, (http://repositorio.cepal.org/bitstream/handle/11362/39855/ S1501295_en.pdf;jsessionid=926CAE33BDD 790D24CEFD2A30013401F sequence $=1$, verificado 25/ $\mathrm{feb} / 2018)$.

Conesa, V. (2010). Guía Metodológica para la Evaluación del Impacto Ambiental. Ediciones Mundiprensa. España.

Departamento Nacional de Planeación (DNP). (2014). Programa Nacional de Adaptación al Cambio Climático (PNACC). ABC: adaptación bases conceptuales marco conceptual y lineamientos del plan nacional de adaptación al cambio climático, Colombia.

Espinoza, G. (2002). Gestión y Fundamentos de Evaluación ambiental. Banco Interamericano de Desarrollo (BID), Centro de Estudios para el Desarrollo (CED). Santiago de Chile.

Garmendia, A; Salvador, A.; Crespo, C.; Garmendia, L. (2005). Evaluación de Impacto ambiental. Editorial PearsonPrentice Hall: Madrid, España.

Grupo Intergubernamental de Expertos sobre el Cambio Climático (IPCC).(2014b). Quinto informe de evaluación del IPCC: Cambio climático. Informe síntesis. Ginebra, Suiza.

Grupo Intergubernamental de Expertos sobre el Cambio Climático (IPCC). (2014ª). Cambio climático, impactos, adaptación y vulnerabilidad. Ginebra, Suiza

Instituto de Hidrología, Meteorología y Estudios Ambientales (IDEAM).; Fondo Financiero de Proyectos de Desarrollo (FONADE). (2013). Efectos del cambio climático en la producción y rendimiento de cultivos por sectores, evaluación del riesgo agroclimático por sectores, Colombia.

Manteiga, L. (2000). Los indicadores ambientales como instrumento para el desarrollo de la política ambiental y su integración en otras políticas. Estadística y medio ambiente, 75-87.

México Resiliente. (2013). Herramientas disponibles en línea de utilidad para evaluar los impactos del cambio climático y apoyar el diseño de medidas de adaptación y mitigación. http://cambioclimatico.conanp.gob.mx/ documentos/Herramientas-para-la-adaptacion.pdf, verificado 12/Nov/2017).

Meza, L. (2014). La agricultura Familiar y el cambio climático. En Salcedo y Guzmán (Ed.) Agricultura Familiar en América Latina. Santiago, Chile: Organización de las Naciones Unidas para la Alimentación y la Agricultura (FAO).

Ministerio de Agricultura y Desarrollo Rural. (2014). Censo Nacional Agropecuario. Inventario Agropecuario. Colombia.

Ministerio de Vivienda, Ordenamiento Territorial y Medio Ambiente del Uruguay. (2016). Evaluación de impacto ambiental. (http://www.mvotma.gub.uy/evaluacion-de-impacto-ambiental,verificado 25/feb/2018). 
Mussetta, P.; Barrientos, M.; Acevedo, E.; Turbay, S.; Ocampo, O. (2016). Vulnerabilidad al cambio climático: Dificultades en el uso de indicadores en dos cuencas de Colombia y Argentina. Revista de Metodología de Ciencias Sociales. 0(36), 119-147. ISSN 1139-5737

Núñez, J. (2005). Los saberes campesinos: implicaciones para una educación rural. Revista Investigacion y Posgrado 19(2), 13-60

Ordaz, J.: Ramírez, D.; Mora, J.; Acosta, A.; Serna, B. (2010). Costa Rica, efectos del cambio climático sobre la agricultura. México, D.F.: CEPAL.

Organización Internacional del Trabajo (OIT), Centro Interamericano para el Desarrollo del Conocimiento en la Formación Profesional (CINTERFOR). (2011). Guía para la evaluación de impactos de formación profesional. Uruguay.

Organización para la Cooperación y el Desarrollo Económico (OECD). (2001). Environmental Indicators. Towards sustainable Development. (http://www.oecd.org/site/worldforum/33703867.pdf, verificado 25/feb/2018).

Peralvo, M.; Postigo, J.; López, S. (2010). Adaptación en Sistemas Productivos Andinos a los efectos del Cambio Climático: revisión y síntesis del estado del conocimiento. Serie Panorama Andino sobre Cambio Climático. CONDESAN, SGCAN. Lima-Quito

Prevochtchikova, M. (2013). La evaluación del impacto ambiental y la importancia de los indicadores ambientales. Revista Gestión y politica pública, México, 22(2), 283-312. ISSN 1405-1079.

Ramírez, L.; Alvarado, A.; Pujol, R.; Mchugh, A.; Brenes, L. (2008). Indicadores para estimar la sostenibilidad agrícola de la cuenca media del río Reventado, Cartago, Costa Rica. Revista Agronomía Costarricense, 32(2), 93-118.

Rave, M. (2016). Incorporación de la Gestión del cambio climático en los planes de desarrollo territorial. Caso de estudio: Eco región eje cafetalero. Tesis de Maestría publicada. Universidad Tecnológica de Pereira. Pereira, Colombia.

Robles, H. (2016). La pequeña agricultura campesina y familiar: construyendo una propuesta desde la sociedad. Entre Diversidades. Revista de Ciencias Sociales y Humanidades. 7, 46-83.

Secretaría de Agricultura, Ganadería, Desarrollo Rural, Pesca y Alimentación (SAGARPA). (2016). Indicadores de cambio climático. México. (http://www.sagarpa.gob.mx/desarrolloRural/Documents/cambioclimatico/ Indicadores\%20agroambienta les.pdf, verificado 25/nov/2017).

Yépez, A. (2012). Cambio Climático: estrategias de gestión con el tiempo en contra... Revista Orinoquia. Universidad de los Llanos, Villavicencio, Meta. Colombia 16(1),77-92. 